Case Report

\title{
Central Venous Catheter-Related Bloodstream Infection with Kocuria kristinae in a Patient with Propionic Acidemia
}

\author{
Masato Kimura, Eichiro Kawai, Hisao Yaoita, Natsuko Ichinoi, \\ Osamu Sakamoto, and Shigeo Kure \\ Department of Pediatrics, Tohoku University Graduate School of Medicine, Sendai, Miyagi 980-8574, Japan \\ Correspondence should be addressed to Masato Kimura; mkimura774@med.tohoku.ac.jp
}

Received 12 July 2016; Revised 26 December 2016; Accepted 4 January 2017; Published 17 January 2017

Academic Editor: Alexandre Rodrigues Marra

Copyright (c) 2017 Masato Kimura et al. This is an open access article distributed under the Creative Commons Attribution License, which permits unrestricted use, distribution, and reproduction in any medium, provided the original work is properly cited.

\begin{abstract}
Kocuria kristinae is a catalase-positive, coagulase-negative, Gram-positive coccus found in the environment and in normal skin and mucosa in humans; however, it is rarely isolated from clinical specimens and is considered a nonpathogenic bacterium. We describe a case of catheter-related bacteremia due to K. kristinae in a young adult with propionic acidemia undergoing periodic hemodialysis. The patient had a central venous catheter implanted for total parenteral nutrition approximately 6 months prior to the onset of symptoms because of repeated acute pancreatitis. K. kristinae was isolated from two sets of blood cultures collected from the catheter. Vancomycin followed by cefazolin for 16 days and 5-day ethanol lock therapy successfully eradicated the $K$. kristinae bacteremia. Although human infections with this organism appear to be rare and are sometimes considered to result from contamination, physicians should not underestimate its significance when it is isolated in clinical specimens.
\end{abstract}

\section{Introduction}

Patients with indwelling foreign materials are known to be at considerable risk of bloodstream infections. Early diagnoses and effective treatments for intravascular catheter-related infections are therefore crucial. Kocuria species are widely distributed in nature and can be found in normal skin and among oral cavity flora in humans and other animals $[1,2] . K$. kristinae was first described in 1974 [3] and is an uncommon pathogenic organism in humans. However, some case reports indicate its emergence as a significant human pathogen [4-8]. We report a case of central venous catheter-related bacteremia with $K$. kristinae and a review of the literature.

\section{Case Report}

A 31-year-old Japanese man with propionic acidemia undergoing total parenteral nutrition presented with a high fever $\left(39.5^{\circ} \mathrm{C}\right)$ after routine hemodialysis at his regional hospital. Although laboratory investigations revealed a slight elevation $(1.2 \mathrm{mg} / \mathrm{dL})$ of C-reactive protein (CRP), he was referred to our university hospital because Gram-positive cocci were detected in blood cultures from the dialysis circuit. He had been undergoing periodic hemodialysis three times a week for 4 years and had received total parenteral nutrition (TPN) for 6 months because of repeated acute pancreatitis.

The patient was originally diagnosed with propionic acidemia following plasma and urinary amino acid analysis in the neonatal period. He developed normally after commencement of a low-protein diet supplemented with Lcarnitine, but he has mild mental retardation. Immunological abnormalities were not diagnosed until admission. At the age of 27 years, he developed cardiomyopathy with a low ejection fraction $(\mathrm{EF}=22.2 \%)$ and oliguria and was treated by cardiac resynchronization with defibrillation therapy (CRTD) and hemodialysis (HD). On admission, his height was $1.57 \mathrm{~m}$, weight was $56.5 \mathrm{~kg}$, and body temperature was $36.7^{\circ} \mathrm{C}$, and he was fully conscious. There was no evidence of abnormalities in the cardiovascular and pulmonary systems. Blood samples for culture were obtained from one peripheral venipuncture site and one central venous catheter (CVC). They were analyzed by VITEK ${ }^{\circledR}$ MS system (bioMérieux S.A., Marcy-l'Étoile, France) and both of them were only identified with $K$. kristinae (blood culture from the dialysis circuit was also 
TABLE 1: The results of antimicrobial susceptibility testing.

\begin{tabular}{lr}
\hline Antimicrobial agent & MIC (mg/L) \\
\hline Ampicillin & $\leq 0.12$ \\
Cefazolin & $\leq 2$ \\
Cefotiam & $\leq 2$ \\
Cefpirome & $\leq 2$ \\
Clindamycin & $\leq 0.5$ \\
Erythromycin & $\leq 0.25$ \\
Gentamicin & $\leq 1$ \\
Imipenem/cilastatin & $\leq 1$ \\
Levofloxacin & 1 \\
Teicoplanin & $\leq 2$ \\
Vancomycin & 1 \\
\hline
\end{tabular}

MIC, minimum inhibitory concentration.

identified with $K$. kristinae in regional hospital). Laboratory data were normal except for increased CRP levels $(4.4 \mathrm{mg} / \mathrm{dL})$. The CVC was left in place, antibiotic therapy with vancomycin was started, and the CVC was locked with ethanol for 5 days. Vancomycin was changed to cefazolin after K. kristinae was found in both blood cultures and its susceptibility to antibiotics was reported (Table 1). Antibiotics were administered for 16 days and the patient was discharged without complications after two serial negative blood cultures from CVC on another day. There was no reinfection after 3 months without antibiotic medication.

\section{Microbiology}

Two sets of blood cultures were obtained from one peripheral venipuncture site and one CVC. The blood samples were added into BacT/Alert ${ }^{\circledR}$ FA Plus and FN Plus resin bottles (bioMérieux S.A., Marcy-l'Étoile, France) and cultured BacT/Alert 3D automated microbial detection systems (bioMérieux S.A., Marcy-l'Étoile, France). Specimens were cultured on chocolate agar plate and were processed by VITEK MS system (bioMérieux S.A., Marcy-l'Étoile, France). VITEK MS is an automated microbial identification system that uses matrix assisted laser desorption ionization timeof-flight mass spectrometry (MALDI-TOF MS) technology to provide identification of microorganisms. Antimicrobial susceptibility tests are performed by Microscan WalkAway 96 Plus System (Beckman Coulter, Inc., CA, USA) and used Microscan Pos MIC 3.3 J panel (Beckman Coulter, Inc., CA, USA). Final molecular identification of microorganism was not carried out.

\section{Discussion}

Kocuria species are catalase-positive, coagulase-negative, Gram-positive coccoid actinobacteria belonging to the Micrococcus family. To date, twenty-two species have been classified in the genus: K. kristinae, K. rosea, K. varians, K. palustris, K. rhizophila, K. marina, K. aegyptia, and others $[1,2]$. They are widely distributed in nature and have been found in normal skin and among oral cavity flora in humans and other animals. K. kristinae was first described by Kloos et al. in 1974 [3] and was not considered to be a human pathogen until about a decade ago. However, some case reports indicate the emergence of $K$. kristinae as a significant human pathogen, mostly in immunocompromised hosts [4], in patients with continuous ambulatory peritoneal dialysis (CAPD) [5], and in central venous catheter infections [4, 6-8].

We reviewed the literature on central venous catheterrelated bloodstream infections (CRBSIs) with $K$. kristinae from 1974 to 2015. We identified 14 cases (including 7 nosocomial infections) and investigated the clinical backgrounds (Table 2 [4, 6-8]). Apart from hospital-acquired infections, there was female predominance (males, 2; females, 5) and the mean age was 44 years (range: $2-89$ ). Long-term catheters are reported to have a lower risk of bloodstream infections than short-term catheters and almost all catheters were longterm types (long-term, 6; peripheral, 1) [9]. The revised guidelines from the IDSA (Infectious Diseases Society of America) published in 2011 recommend combination therapy with antibiotic administration through the colonized catheter for 10-14 days and antibiotic lock therapy in cases of uncomplicated CRBSI [10]. A review of the literature indicated that only one case was treated with combination therapy and the catheter was finally removed [4]. To the best of our knowledge, our patient represents the first successful treatment using combination therapy with antibiotics and antibiotic lock therapy to preserve the central venous catheter.

In conclusion, we have described a case of K. kristinae bacteremia in a patient with a central venous catheter and reviewed the literature. Although K. kristinae infections in humans appear to be rare and the organism was previously considered harmless, medical practitioners should be aware of the significance of this little recognized pathogen when it is isolated in clinical specimens.

\section{Competing Interests}

The authors declare that there are no competing interests regarding the publication of this paper. 


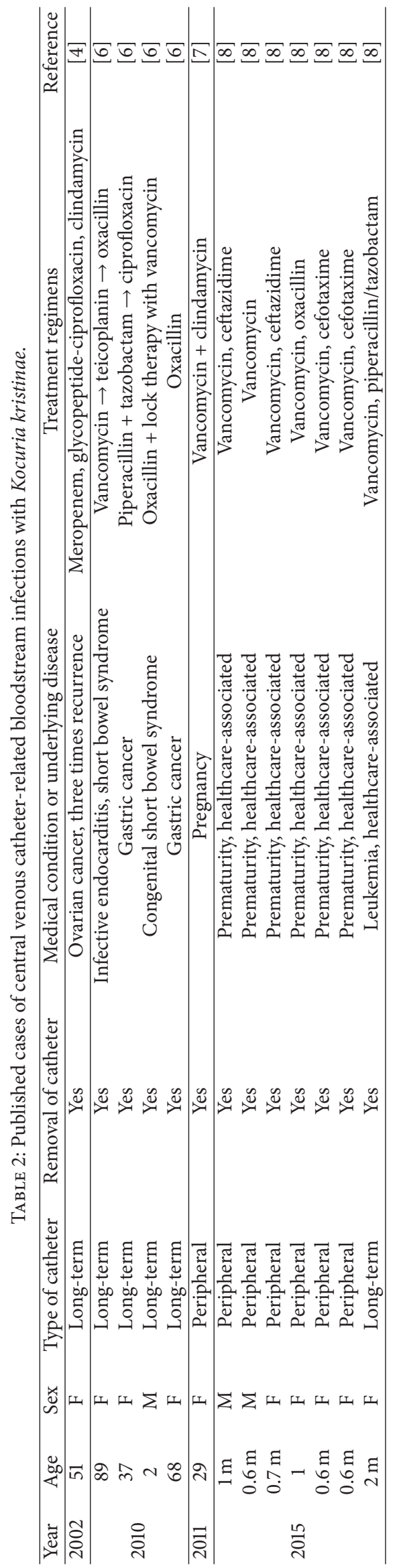




\section{References}

[1] E. Stackebrandt, C. Koch, O. Gvozdiak, and P. Schumann, "Taxonomic dissection of the genus Micrococcus: Kocuria gen. nov., Nesterenkonia gen. nov., Kytococcus gen. nov., Dermacoccus gen. nov., and Micrococcus cohn 1872 gen. emend," International Journal of Systematic Bacteriology, vol. 45, no. 4, pp. 682692, 1995.

[2] V. Savini, C. Catavitello, G. Masciarelli et al., "Drug sensitivity and clinical impact of members of the genus Kocuria," Journal of Medical Microbiology, vol. 59, no. 12, pp. 1395-1402, 2010.

[3] W. E. Kloos, T. G. Tornabene, and K. H. Schleifer, "Isolation and characterization of micrococci from human skin, including two new species: Micrococcus lylae and Micrococcus kristinae," International Journal of Systematic Bacteriology, vol. 24, no. 1, pp. 79-101, 1974.

[4] G. Basaglia, E. Carretto, D. Barbarini et al., "Catheter-related bacteremia due to Kocuria kristinae in a patient with ovarian cancer," Journal of Clinical Microbiology, vol. 40, no. 1, pp. 311313, 2002.

[5] A. Carlini, R. Mattei, I. Lucarotti, A. Bartelloni, and A. Rosati, "Kocuria kristinae: an unusual cause of acute peritoneal dialysis-related infection," Peritoneal Dialysis International, vol. 31, no. 1, pp. 105-107, 2011.

[6] C. C. Lai, J. Y. Wang, S. H. Lin et al., "Catheter-related bacteraemia and infective endocarditis caused by Kocuria species," Clinical Microbiology and Infection, vol. 17, no. 2, pp. 190-192, 2011.

[7] R. Dunn, S. Bares, and M. Z. David, "Central venous catheterrelated bacteremia caused by Kocuria kristinae: case report and review of the literature," Annals of Clinical Microbiology and Antimicrobials, vol. 10, article 31, 2011.

[8] H.-M. Chen, H. Chi, N.-C. Chiu, and F.-Y. Huang, "Kocuria kristinae: a true pathogen in pediatric patients," Journal of Microbiology, Immunology and Infection, vol. 48, no. 1, pp. 8084, 2015.

[9] D. G. Maki, D. M. Kluger, and C. J. Crnich, "The risk of bloodstream infection in adults with different intravascular devices: a systematic review of 200 published prospective studies," Mayo Clinic Proceedings, vol. 81, no. 9, pp. 1159-1171, 2006.

[10] N. P. O'Grady, M. Alexander, L. A. Burns et al., "Guidelines for the prevention of intravascular catheter-related infections," American Journal of Infection Control, vol. 39, no. 4, pp. S1-S34, 2011. 


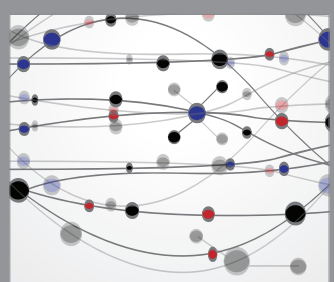

The Scientific World Journal
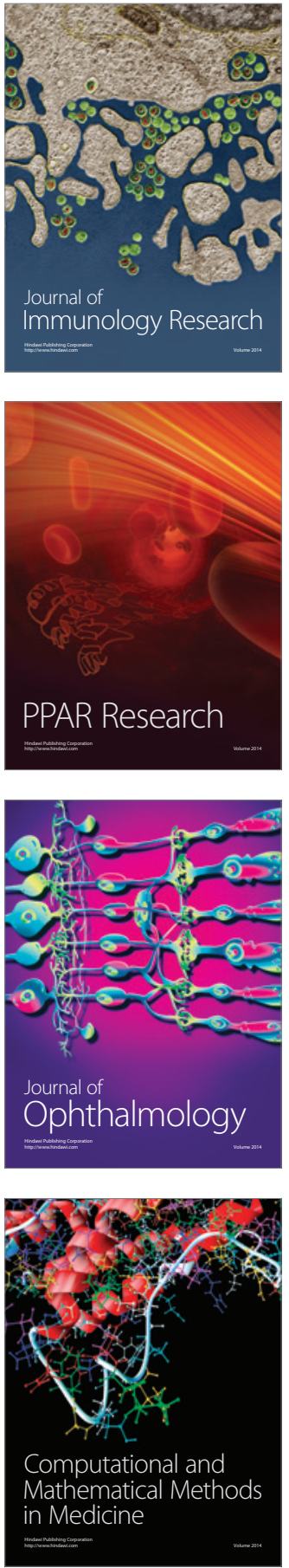

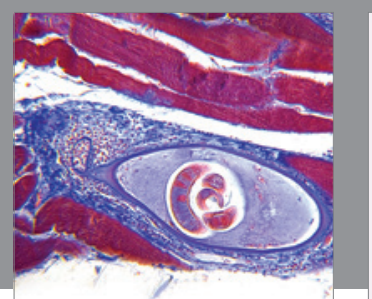

Gastroenterology Research and Practice
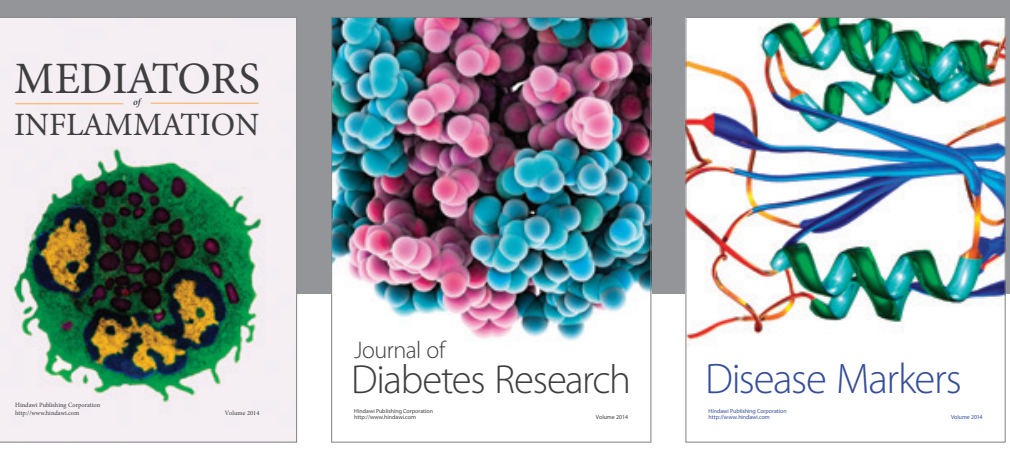

Disease Markers

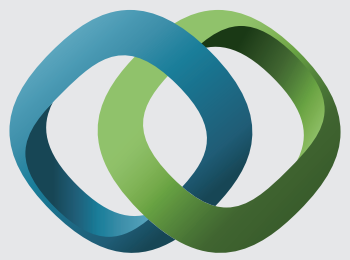

\section{Hindawi}

Submit your manuscripts at

https://www.hindawi.com
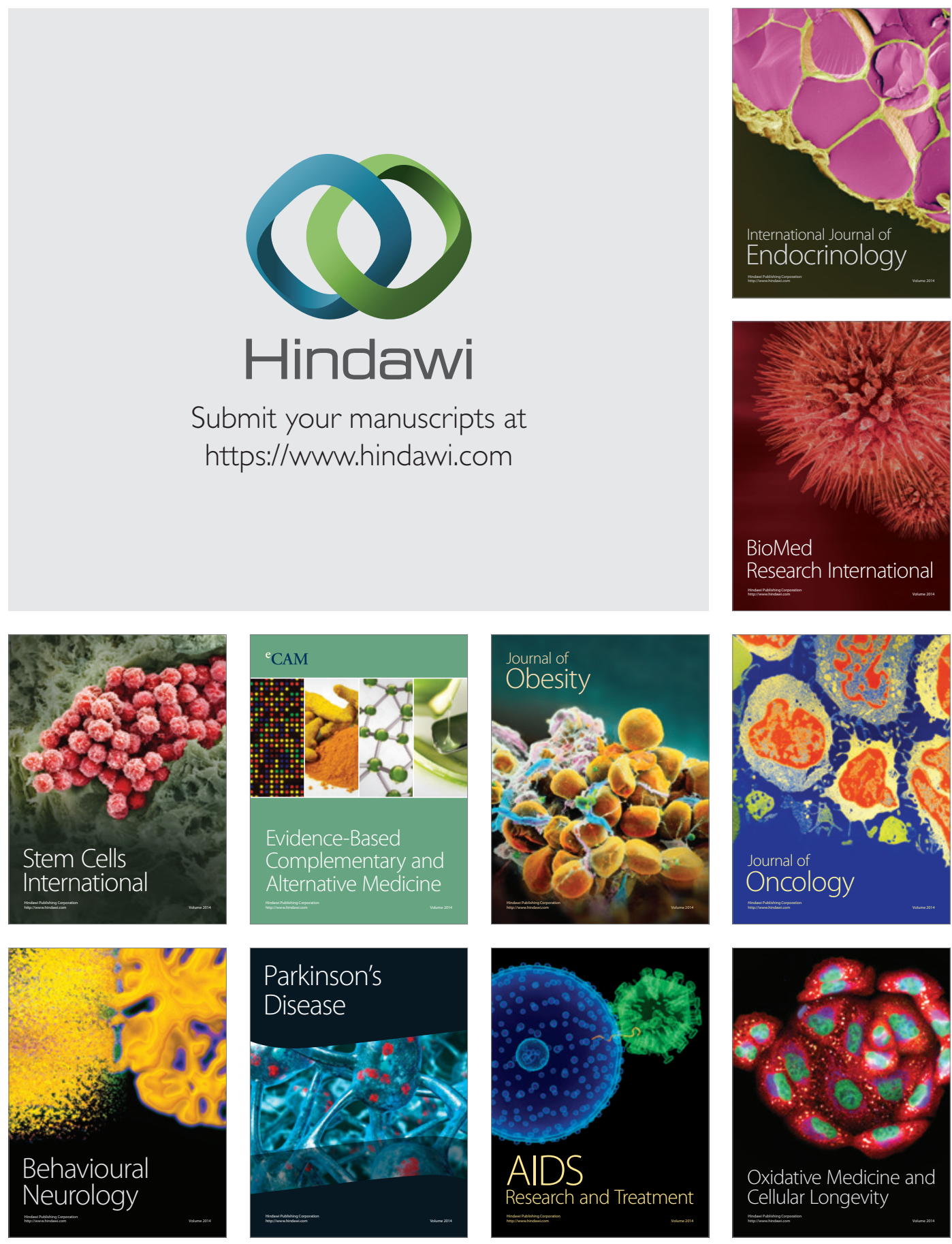